\title{
Analysis of the Effective and Internal Cyclic Stress Components in the Inconel Superalloy Fatigued at Elevated Temperature
}

\section{ŠMíD Miroslav ${ }^{\mathrm{a}}$, PETRENEC Martin ${ }^{\mathrm{b}}$, POLÁK Jaroslav ${ }^{\mathrm{c}}$ OBRTLÍK Karel ${ }^{\mathrm{d}}$, CHLUPOVÁ Alice ${ }^{\mathrm{e}}$}

\author{
Institute of Physics of Materials, AS CR, Žižkova 22, Brno 616 62, Czech Republic \\ asmid@ipm.cz, ${ }^{\mathrm{b}}$ petrenec@ipm.cz, cpolak@ipm.cz, ${ }^{\mathrm{a}}$ prasil@ipm.cz
}

\begin{abstract}
Keywords: Low cycle fatigue, superalloys, high temperature, hysteresis loop, effective and internal stresses.

Abstract. Cyclic multiple step test in strain control have been performed on cylindrical specimens of cast polycrystalline Inconel 738LC and 792-5A superalloys at $800{ }^{\circ} \mathrm{C}$ in laboratory atmosphere. Hysteresis loops were analyzed according to the statistical theory of hysteresis loop. The effective and internal stress components were evaluated. The effective stress of $\gamma^{\prime}$ precipitate has significant influence on the stress-strain response both materials. The stress amplitude in IN 792-5A is higher than in IN 738LC at approximately same total strain amplitude due to significantly higher effective stress of $\gamma^{\prime}$ phase. Cyclic hardening/softening curves and cyclic stress-strain curves using short-cut procedure were obtained. Cyclic hardening/softening behavior depends both on temperature and strain amplitude. Low amplitude straining is characterized by the saturation of the stress amplitude. In high amplitude straining slight softening was found. The cyclic stress-strain curves for both materials can be fitted by power law. Cyclic stress-strain response in terms of internal and effective stress components is discussed in relation to microstructural parameters of the materials. The observation of surface relief revealed the presence of persistent slip markings.
\end{abstract}

\section{Introduction}

Precipitation strengthened nickel base superalloys exhibits excellent high temperature strength and hot corrosion resistance and therefore they are used for production of blades and discs of gas turbine engines [1]. The critical turbine parts are subjected elastic-plastic straining due to repeated loading and thermal gradients during start-up and shut-down periods. Thus, it is important to study low cycle fatigue properties of these materials. Structure of nickel base superalloys generally consist of two components, matrix $\gamma$ and precipitate $\gamma^{\prime}$ ( $\mathrm{Ll}_{2}$ type structure) with different mechanical properties. Analysis of hysteresis loops shape can provide important information about the sources of the high cyclic stress in such complex materials. The aim of this paper was to conduct cyclic test at $800^{\circ} \mathrm{C}$ of two commonly used cast polycrystalline superalloys (IN 738LC and IN 792-5A) and study cyclic stress-strain response of these materials using generalized statistical theory of the hysteresis loop [2].

\section{Experimental}

Inconel 738LC and Inconel 792-5A were provided by PSB Turbo, Velká Bíteš a.s. as conventionally cast polycrystalline rods in fully heat treated condition. Chemical composition of both superalloys is shown in Table 1. Cylindrical button-end specimens were machined parallel to the rod axis with gauge length $15 \mathrm{~mm}$ and diameter $6 \mathrm{~mm}$.

Structure of materials is fully dendritic. In interdendritical areas frequent presence of eutectics $\gamma / \gamma^{\prime}$, carbides and primarily shrinkage pores with size up to $0.7 \mathrm{~mm}$ is observed. Average grain size determined by linear intercept method was around $3 \mathrm{~mm}$ for both materials. Fig. 1 shows typical structure of experimental material. Precipitates $\gamma^{\prime}$ in IN 738LC have nearly cuboidal or irregular shape. Their average diameter was around $670 \mathrm{~nm}[3,4]$. Microstructure of IN 792-5A is more symmetrical and consists of two types of precipitates: cuboidal and small spherical with sizes 480 and $115 \mathrm{~nm}$ respectively [5,6]. TEM study revealed $\gamma^{\prime}$ precipitate volume fraction of $56 \%$ for IN 738LC $[3,4]$ and $68 \%$ in IN 792-5A [5-7]. 
Table 1. Chemical composition of experimental superalloys [wt. \%].

\begin{tabular}{|c||c|c|c|c|c|c|c|c|c|c|c|c|c|}
\hline & $\mathrm{Cr}$ & $\mathrm{Co}$ & $\mathrm{Ti}$ & $\mathrm{Al}$ & $\mathrm{Ta}$ & $\mathrm{W}$ & $\mathrm{Mo}$ & $\mathrm{Nb}$ & $\mathrm{Fe}$ & $\mathrm{Zr}$ & $\mathrm{C}$ & $\mathrm{B}$ & $\mathrm{Ni}$ \\
\hline IN 738LC & 16.22 & 8.78 & 3.37 & 3.35 & 1.77 & 2.63 & 1.71 & 0.84 & 0.2 & 0.04 & 0.1 & 0.008 & rest \\
\hline IN 792-5A & 12.28 & 8.87 & 3.98 & 3.36 & 4.12 & 4.1 & 1.81 & 0.1 & 0.16 & 0.031 & 0.078 & 0.015 & rest \\
\hline
\end{tabular}

b)
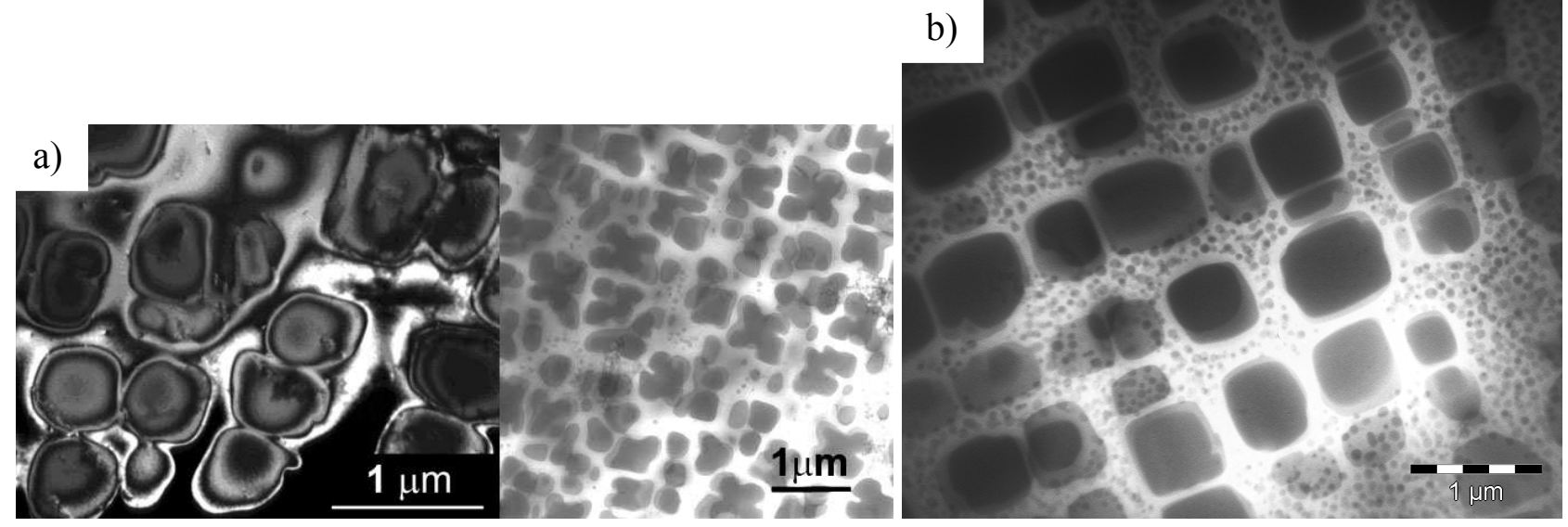

Fig. 1. The microstructure of two superalloys a) IN 738LC - TEM and SEM in transmission mode micrographs b) IN 792-5A - TEM micrograph.

Specimens were cyclically strained in a computer controlled electro-hydraulic MTS testing system at total strain rate $2 \times 10^{-3} \mathrm{~s}^{-1}$ with fully reversed total strain cycle $\left(\mathrm{R}_{\varepsilon}=-1\right)$. Sensitive extensometer with $12 \mathrm{~mm}$ base was used for strain control and measurement. The tests were conducted at temperature $800{ }^{\circ} \mathrm{C}$ in air. Multiple step test method [2] was used for obtaining cyclic stress-strain curve, therefore only one specimen was needed to obtain cyclic stress-strain curve. Data recording rate was under $0.3 \mathrm{~ms}$ in order to record high number of data on the hysteresis loop. Using maximum and minimum strains in cycles, the relative strain $\varepsilon_{\mathrm{r}}$ and the relative stress $\sigma_{\mathrm{r}}$ [2] were calculated for the tensile and for the compressive hysteresis half-loops. The first and the second derivatives of the hysteresis half-loops were obtained using smoothing numerical procedures. For each point of the first and the second derivatives the number of the neighbour points involved in their determination could be chosen.

For the surface relief observations the specimens were before cycling mechanically and electrolytically polished. Observations were conducted using SEM JEOL JSM6460 and high resolution field emission scanning electron microscope MIRA 3 FEG-SEM from Tescan Co.

\section{Results and discussion}

Cyclic stress-strain response: Fig. 2a shows the dependence of the stress amplitude $\sigma_{a}$ on number of cycles $N$ during the test at increasing total strain amplitudes. Superalloy IN 792-5A exhibits stabilized stress response at low and also at high strain amplitudes. The stabilized stress response was also observed during cyclic straining of superalloy IN 738LC at low amplitudes, but at amplitudes higher than $\varepsilon_{\mathrm{a}}=0.35 \%$ slight cyclic softening appeared. The strain amplitudes in testing both materials were the approximately the same and it is clearly visible that stress response of IN 792-5A is significantly higher than that of IN 738LC. The main reason for this difference is different elastic moduli of individual samples (see Table 2, for short-cut method). It could be also related to different microstructures of both materials. While IN 738LC is composed from one type of precipitates, IN 792-5A has two types, one of them are fine precipitates in the matrix which make the movement of dislocations more difficult. Beneficial for higher stress response of this material is also higher volume fraction of $\gamma^{\prime}$ and more aligned structure. 


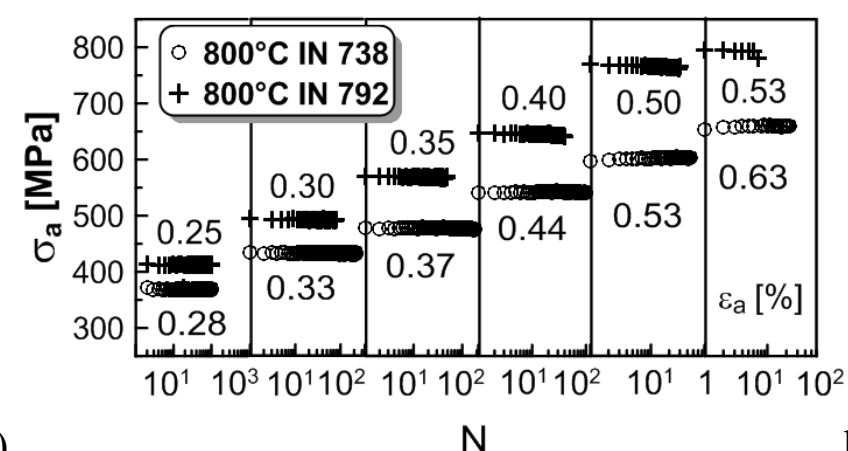

b)

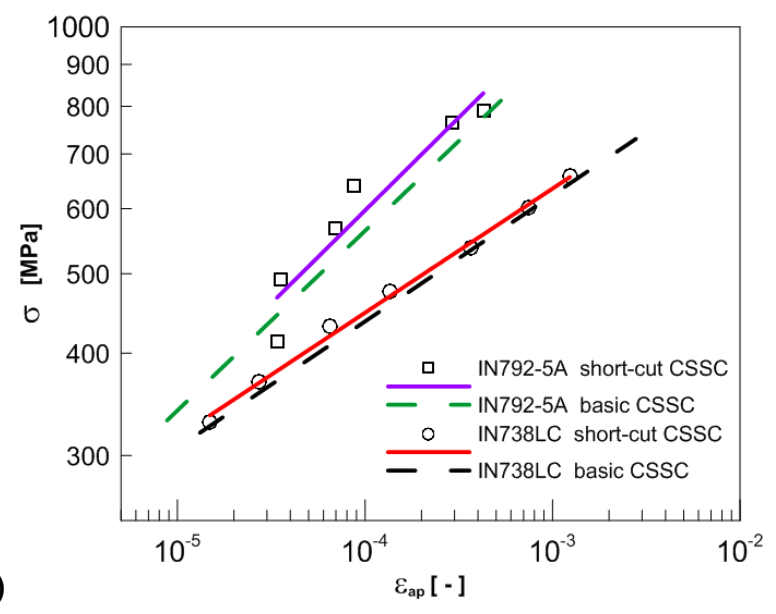

Fig. 2. a) Cyclic hardening/softening curves, b) Basic cyclic stress-strain curves and those obtained using short-cut procedure for both superalloys at $800{ }^{\circ} \mathrm{C}$.

Table 2. The parameters of CSSCs and elastic moduli of both superalloys at $800^{\circ} \mathrm{C}$.

\begin{tabular}{|c||c|c|c|c|c|c|}
\hline \multirow{2}{*}{} & \multicolumn{3}{|c|}{ short-cut CSSC } & \multicolumn{3}{c|}{ basic CSSC [3] } \\
\cline { 2 - 7 } & $K^{\prime}$ & $n^{\prime}$ & $E$ & $K^{\prime}$ & $n^{\prime}$ & $E$ \\
\cline { 2 - 7 } & {$[\mathrm{MPa}]$} & {$[-]$} & {$[\mathrm{GPa}]$} & {$[\mathrm{MPa}]$} & {$[-]$} & {$[\mathrm{GPa}]$} \\
\hline \hline IN 738LC & 1840 & 0.158 & 130 & 1808 & 0.154 & 130 \\
\hline IN 792-5A & 4241 & 0.219 & 168 & 4492 & 0.224 & 170 \\
\hline
\end{tabular}

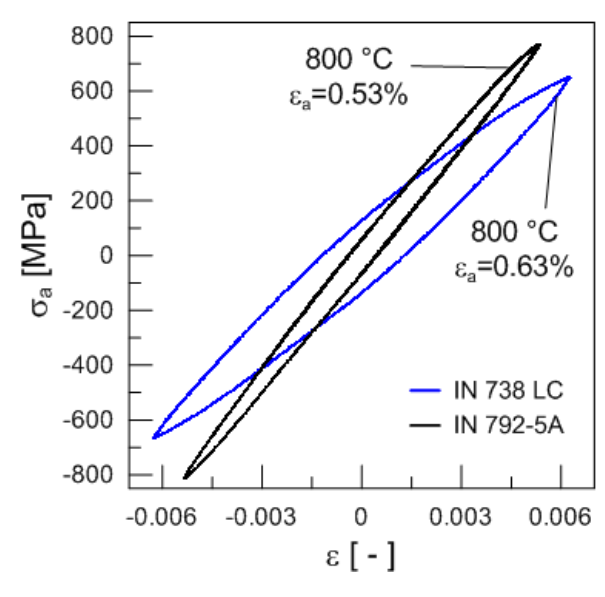

Fig. 3. Saturated hysteresis loops for the highest strain amplitudes.

Points in Fig. $2 b$ were calculated as the average value of stress and plastic strain amplitudes of a few half loops at the end of each straining block and they represent the cyclic stress-strain curves (CSSCs) obtained using short-cut method. Experimental data were approximated by the power law in the form $\sigma_{a}=K^{\prime}\left(\varepsilon_{a p}\right)^{n^{\prime}}$ where $K^{\prime}$ is fatigue hardening coefficient and $n^{\prime}$ fatigue hardening exponent. These two parameters were evaluated using linear regression analysis and are shown in Table 2. Full lines represent fitted line to experimental points from short-cut method and dashed lines represent the basic CSSCs [3] displayed for comparison. The short-cut CSSCs are in satisfactory agreement with basic ones showing that short-cut method yields reasonable results. It is apparent from Fig. 2b that the cyclic stress-strain curve of IN 792-5A is above that of IN 792-5A (see also saturated hysteresis loops in Fig. 3). 

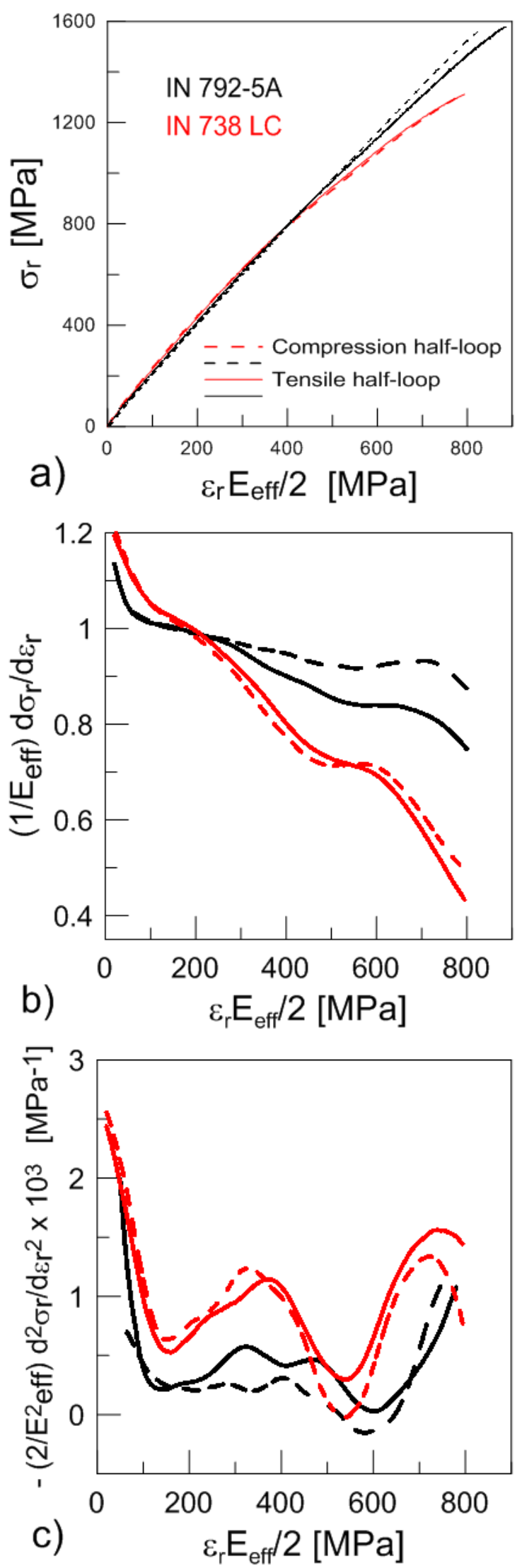

Fig. 4. a) The saturated hysteresis halfloops with their b) the first and c) the second derivatives of both superalloys at $800{ }^{\circ} \mathrm{C}$.
Hysteresis loop analysis: Saturated hysteresis loops obtained from the last step of multiple step tests of both superalloys (IN 738LC cycled with $\varepsilon_{\mathrm{a}}=0.63 \%$ and IN 792-5A cycled with $\varepsilon_{\mathrm{a}}=0.53 \%$ ) were recorded at $800{ }^{\circ} \mathrm{C}$ (Fig. 3). The plastic strain amplitudes are $\varepsilon_{\mathrm{ap}}=0.12 \%$ for IN 738 and $\varepsilon_{\mathrm{ap}}=$ $0.044 \%$ for IN 792-5A. Fig. 4a shows the tensile and compression hysteresis half-loops plotted in relative stress $\sigma_{\mathrm{r}}$ vs. fictive stress $\varepsilon_{\mathrm{r}} E_{\mathrm{eff}} / 2$ [2]. The half of the relative strain $\varepsilon_{\mathrm{r}}$ multiplied by compression or tension effective modulus (see Table 3) are used for the calculation of the fictive stress. The shape of the halfloops was analyzed by plotting the first and the second derivatives of tensile or compression halfloops (divided by effective modulus $E_{\text {eff }}$ and by the half of the square of effective modulus) vs. fictive stress (Figs 4b and 4c) [2,4]. Both effective moduli $E_{\text {eff }}$ are found from the first derivative at relative strain where the second derivative reaches its first minimum. The initial drop of the second derivative until the first minimum corresponds to the relaxation of the plastic strain under decreasing effective stress. The plots of the first and the second derivatives of both two-phase superalloys with phases having different critical internal stresses are displayed for $800{ }^{\circ} \mathrm{C}$ in Figs $4 \mathrm{~b}$ and $4 \mathrm{c}$. In IN 738 two peaks of the second derivative that approximate the probability density function (PDF) of the critical internal stresses are present. They correspond to the subsequent plastic deformation of $\gamma$ and $\gamma^{\prime}$ phases within a cycle. It was shown earlier [4] that the second peak changes with increasing number of loading cycles. The effective stresses $\sigma_{\text {eff }}$ (see Table 3 ) of both phases were estimated from the relative strain corresponding to the onset of respective peak of probability density function of the internal critical stresses. The analysis of the hysteresis half-loop of IN 792-5A (Fig. 4c) reveals only two small poorly defined first maxima. This is due to the presence of small rounded precipitates which are embedded in the matrix. The beginning of the second maximum shifted to higher fictive stresses in comparison with IN 738 is evident. The effective stresses of $\gamma$ and $\gamma^{\prime}$ phases in IN792-5A can be estimated and are shown in Table 3. The effective stress in $\gamma^{\prime}$ phase is high and is in agreement with the difficult movement of dislocations in an ordered structure.

The effective stress of the $\gamma^{\prime}$ phase in IN792-5A is higher than in IN738LC which it can be connected with different chemical composition and volume fraction of $\gamma^{\prime}$ precipitate. 
Previous TEM study of the internal dislocation structure in Inconel 713LC superalloy [8] and in IN 792-5A alloy [9] revealed localized cyclic plastic straining in the planar bands parallel to $\{111\}$ planes up to temperature $900{ }^{\circ} \mathrm{C}$. The stress components thus correspond to the dislocation arrangement and dislocation velocities in the bands of intensive glide. In high temperature straining the continuous recovery of the $\gamma$ phase leads to stabilized stress response. The parallel planar bands can be observed at the surface of both specimens.

Table 3. Effective elastic moduli and effective stresses of two phases of both superalloys at $800{ }^{\circ} \mathrm{C}$.

\begin{tabular}{|c|c||c|c|c|}
\hline Superalloy & Half-loop & $\begin{array}{c}\mathrm{E}_{\text {eff }} \\
{[\mathrm{GPa}]}\end{array}$ & $\begin{array}{c}\text { Matrix } \\
\sigma_{\text {eff }}[\mathrm{MPa}]\end{array}$ & $\begin{array}{c}\text { Precipitates } \\
\sigma_{\text {eff }}[\mathrm{MPa}]\end{array}$ \\
\hline \multirow{2}{*}{ IN 738LC } & tensile & 129 & 162 & 547 \\
\cline { 2 - 5 } & compression & 130 & 153 & 541 \\
\hline \multirow{2}{*}{ IN 795-5A } & tensile & 140 & 158 & 643 \\
\cline { 2 - 5 } & compression & 145 & 126 & 579 \\
\hline
\end{tabular}

Surface relief observation: Selected specimens from both materials were cycled to fracture and their surface was studied using the scanning electron microscope. The samples of both materials were cycled with approximately the same total strain amplitude. The plastic strain amplitude for IN 738LC was $0.14 \%$ and for IN 792-5A $0.37 \%$. Fig. 5a, b show characteristic surface relief. Slip markings of IN 738LC specimen consist of extrusions and parallel intrusions. Slip markings consisting only from extrusions were present on IN 792-5A specimen. Markings of different slip systems were observed in grains suitably oriented to stress axis and they often run along the whole grain. The observation of the surface relief proves that cyclic plastic strain is highly localized into persistent slip bands, which are thin and pass through both the $\gamma$ matrix and $\gamma$ ' precipitates. As a result slip markings, or persistent slip markings are produced on the surface of individual grains. They consist preferentially of parallel extrusions with density depending on the superalloy and plastics strain amplitude. The width and the height of the extrusions was larger at high temperature than at low temperatures and the extrusions were accompanied by parallel intrusions. Presumably, extrusions are present at all temperatures, however, standard SEM techniques cannot identify them [10]. Due to localization of the cyclic plastic strain the stress-strain response of IN 738LC superalloy reflects the cyclic straining only in localized volume with local plastic strain more than an order of magnitude higher than the applied plastic strain amplitude. Nevertheless, macroscopic stress-strain response reflects both the local stress-strain behaviour and the degree of cyclic strain localization.

a)

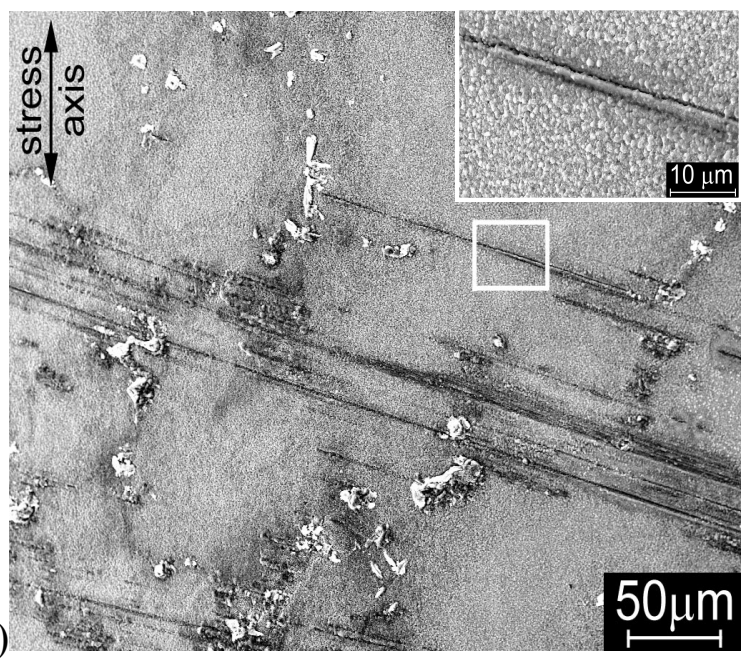

b)

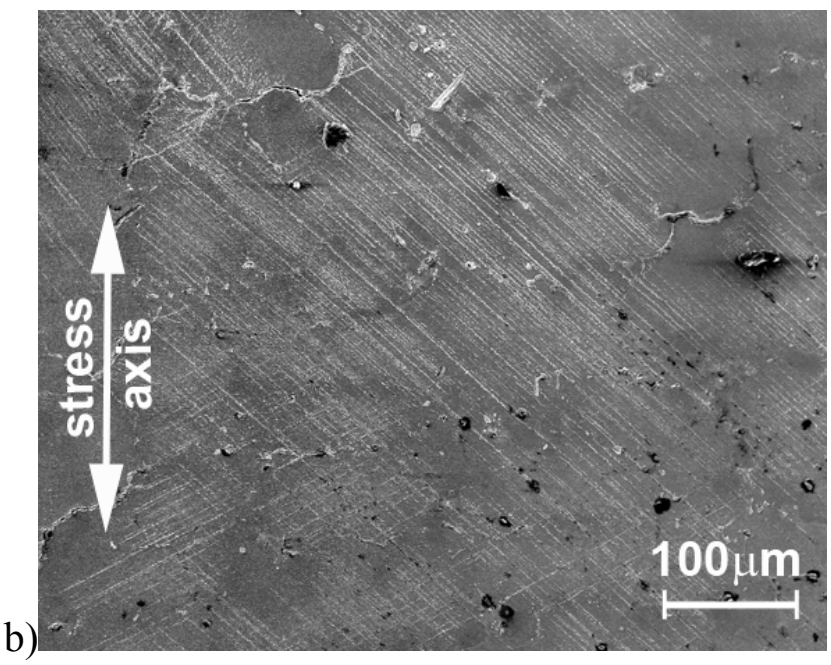

Fig. 5. SEM micrographs of surface relief of specimens cycled to fracture at $800^{\circ} \mathrm{C}$ : a) IN $738 \mathrm{LC}$ $\left.\left(\varepsilon_{\mathrm{a}}=0.5 \%, N_{\mathrm{f}}=240\right) ; \mathrm{b}\right)$ IN 792-5A $\left(\varepsilon_{\mathrm{a}}=0.51 \%, N_{\mathrm{f}}=147\right)$. 


\section{Conclusions}

The results of total strain controlled fatigue tests in symmetrical strain cycling and the observations of surface relief produced by cyclic straining in cast Inconel 738LC and 792-5A polycrystals at 800 ${ }^{\circ} \mathrm{C}$ can be summarized as follows:

1. Cyclic straining results in stabilized behaviour in Inconel 792-5A, while cyclic softening is observed in Inconel 792-5A.

2. Cyclic stress-strain curves of both superalloys obtained using short-cut procedure coincides with the basic stress-strain curve. The CSSC of Inconel 792-5A is shifted to higher stress amplitude in comparison with that of Inconel 738LC due to the different quantity and type of precipitates.

3. The analysis of the hysteresis loop based on statistical theory of the hysteresis loop allows estimating the contributions of the effective stresses and the distribution of the critical internal stresses.

4. Cyclic plastic strain is highly localized into persistent slip bands. The macroscopic cyclic stress-strain response reflects the localized stress-strain response and the degree of cyclic strain localization.

5. High stress response of the Inconel 792-5A than that of the Inconel 738LC superalloy is due to higher effective stresses of respective $\gamma^{\prime}$ phases.

\section{Acknowledgements}

This research was supported by the project No. AV0Z 2041057 of the Academy of Sciences of the Czech Republic and the grant 106/08/1631 of the Grant Agency of the Czech Republic. The authors are also obliged to Mr. Dluhoš for SEM observations using TESCAN MIRA 3 microscope.

\section{References}

[1] M.J. Donachie, S.J. Donachie. Superalloys: A Technical Guide. ASM International, Materials Park, 2002.

[2] J. Polák: Cyclic Plasticity and Low Cycle Fatigue Life of Metals. Elsevier, Amsterdam, 1991.

[3] K. Obrtlík, A. Chlupová, M. Petrenec and J. Polák: Key Engineering Mater. Vol. 385-387 (2008), p. 581

[4] M. Petrenec, M. Šmíd, K. Obrtlík, J. Polák, In: Proceedings of 17th European Conference on Fracture. Brno : VUTIUM Brno, (2008), p. 1358-1365.

[5] M. Petrenec, K. Obrtlík, J. Polák and J. Man: Key Engineering Mater. Vol. 345-346 (2007), p. 383

[6] M. Petrenec, K. Obrtlík and J. Polák: Key Engineering Mater. Vol. 348-349 (2007), p. 101

[7] J. Hakl, T. Vlasák, J. Lapin: Metallic Materials Vol. 45 (2007), p. 177

[8] M. Petrenec, K. Obrtlík and J. Polák: Mater. Sci. Engng. Vol. A400-401 (2005), p. 485

[9] M. Petrenec, K. Obrtlík, J. Polák and J. Man: Mater. Sci. Forum Vol. 567-568 (2008), p. 429

[10] J. Polák, J. Man, K. Obrtlík K. and T. Kruml: Z. Metallkd. Vol. 94 (2003), p. 1327 